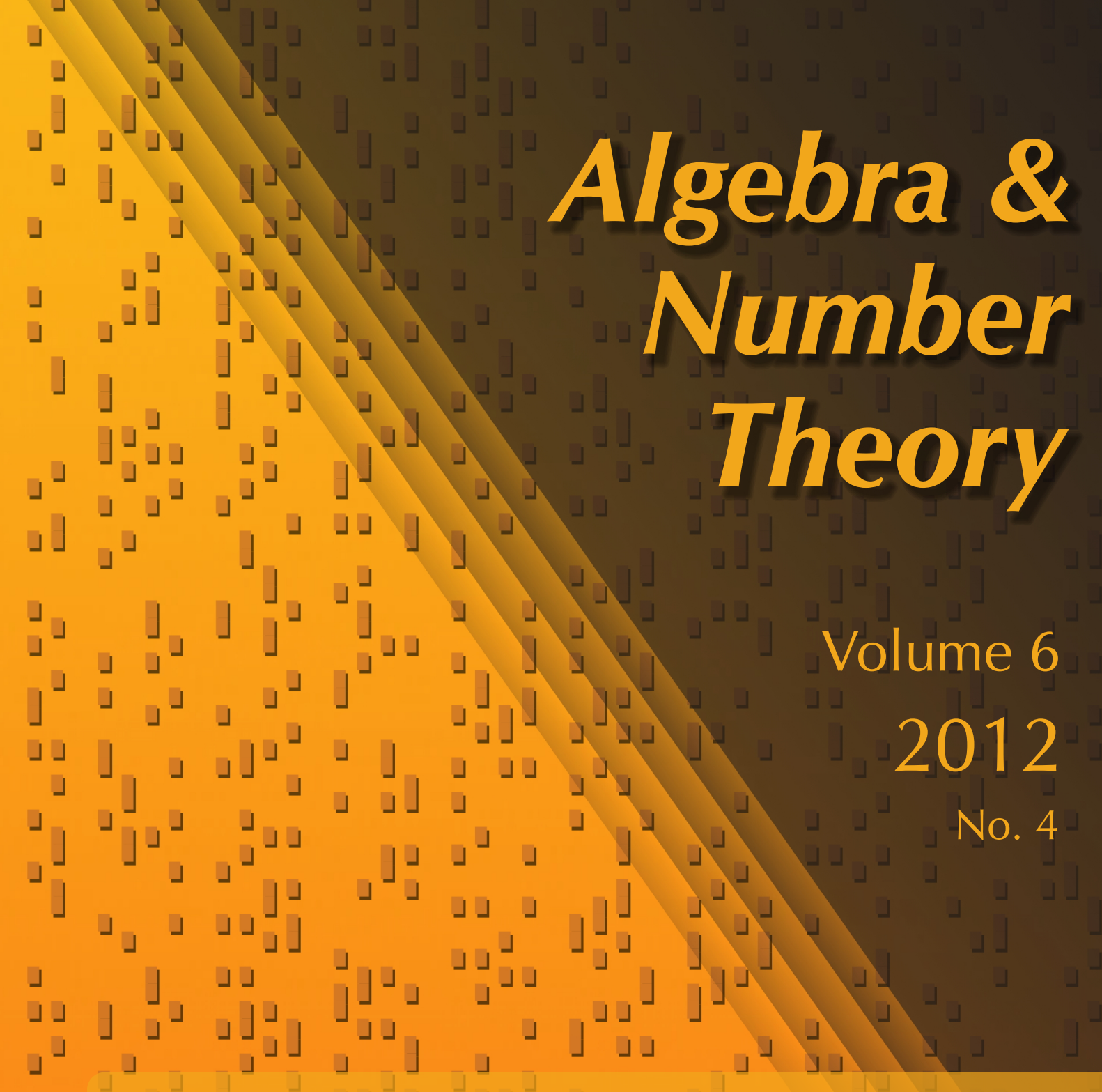

Realizing large gaps in cohomology for symmetric group modules

David J. Hemmer

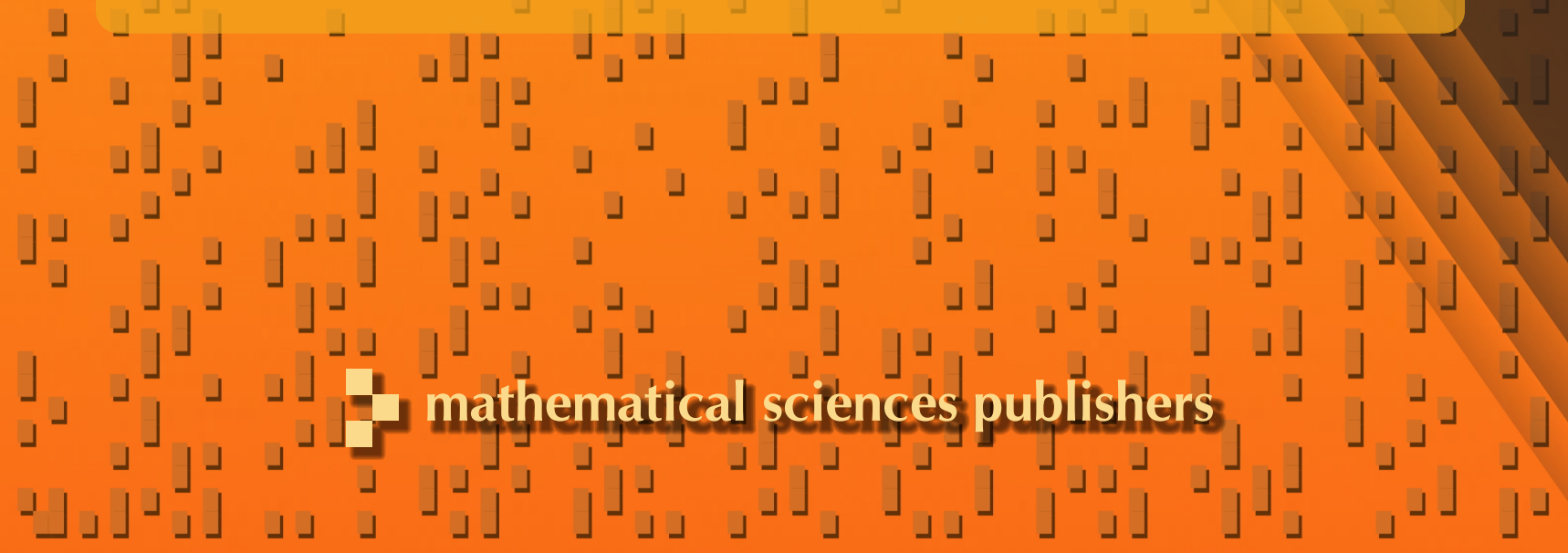




\title{
Realizing large gaps in cohomology for symmetric group modules
}

\author{
David J. Hemmer
}

\begin{abstract}
Using results of the author with Cohen and Nakano, we find examples of Young modules $Y^{\lambda}$ for the symmetric group $\Sigma_{d}$ for which the Tate cohomology $\widehat{\mathrm{H}}^{i}\left(\Sigma_{d}, Y^{\lambda}\right)$ does not vanish identically, but vanishes for approximately $\frac{1}{3} d^{3 / 2}$ consecutive degrees. We conjecture these vanishing ranges are maximal among all $\Sigma_{d}$-modules with nonvanishing cohomology. The best known upper bound on such vanishing ranges stands at $(d-1)^{2}$, due to work of Benson, Carlson and Robinson. Particularly striking, and perhaps counterintuitive, is that these Young modules have maximum possible complexity.
\end{abstract}

\section{Introduction}

Let $G$ be a finite group and $k$ an algebraically closed field of characteristic $p$. If $G$ contains an element $x$ of order $p$ such that the centralizer $C_{G}(x)$ is not $p$-nilpotent, then a result of Benson [1995] guarantees the existence of a nonprojective $k G$ module $M$ in the principal block such that the cohomology $\mathrm{H}^{*}(G, M)$ is identically zero. For the remaining principal block modules, those with nonvanishing cohomology, one might ask for the smallest degree that is nonzero, or the number of consecutive degrees in which the cohomology vanishes. In [Benson et al. 1990], Benson, Carlson and Robinson gave an upper bound $r=r(G)$ on the number of consecutive $i$ for which the cohomology $\mathrm{H}^{i}(G, M)$ can vanish, without being identically zero:

Theorem 1.1 [Benson et al. 1990, Theorem 2.4]. Given a finite group G, there exists a positive integer $r$ such that for any commutative ring $R$ of coefficients and any $R G$-module $M$, if $\hat{\mathrm{H}}^{i}(G, M)=0$ for $r+1$ consecutive values of $i$ then $\hat{\mathrm{H}}^{i}(G, M)=0$ for all $i$ positive and negative.

The $\hat{\mathrm{H}}$ above denotes Tate cohomology, which agrees with the ordinary cohomology in positive degrees. The proof of Theorem 1.1 expresses $r$ in terms of the

Research of the author was supported in part by NSF grant DMS-0808968.

MSC2010: 20C30.

Keywords: symmetric group, cohomology, Young module. 
degrees of a set of homogenous generators for the cohomology ring of $G$. However, there is no expectation that this $r$ should be the best possible bound.

There do not appear to be any examples in the literature demonstrating large gaps in cohomology, or determining the smallest possible value of $r$ for particular groups. This is not surprising, as calculating $\mathrm{H}^{*}(G, M)$ is generally difficult. In [Cohen et al. 2010] the author, with Cohen and Nakano, obtained some very general results when $M$ is a Young module $Y^{\lambda}$ for the symmetric group $\Sigma_{d}$. The goal of this paper is to use these results to find very large gaps in Young module cohomology. For certain partitions $\lambda \vdash d$ in characteristic two, we find the minimal $i \geq 0$ with $\mathrm{H}^{i}\left(\Sigma_{d}, Y^{\lambda}\right) \neq 0$. These gaps turn out to be the largest possible among all Young modules, and come "close" to realizing the value of $r$ arising from Theorem 1.1. Remarkably the Young modules with the largest vanishing ranges also have maximum possible complexity. That is the dimensions in a minimal projective resolution grow as quickly as possible. See [Benson 1998, p.153] for the precise definition of complexity.

\section{Computing Young module cohomology}

In this section we recall results from [Cohen et al. 2010] on computing Young module cohomology. Let $V \cong k^{d}$ be the natural module for the general linear group $G:=\mathrm{GL}_{d}(k)$. For a partition $\lambda \vdash d$, let $L(\lambda)$ denote the simple $G$-module of highest weight $\lambda$, and let $Y^{\lambda}$ denote the Young module for $\Sigma_{d}$. We denote by $\unrhd$ the usual dominance order on partitions of $d$, and by $\lambda^{\prime}$ the transpose or conjugate partition. Definitions and information on all these modules can be found in [Martin 1993].

The commuting actions of $G$ and $\Sigma_{d}$ on $V^{\otimes d}$ give the homology $\mathrm{H}_{i}\left(\Sigma_{d}, V^{\otimes d}\right)$ the structure of a $G$-module. The composition multiplicities of this $G$-module are related to the dimensions of Young module cohomology in the following way. Let $[M: S]$ denotes the multiplicity of a simple module $S$ in a composition series of $M$.

Theorem 2.1 [Doty et al. 2004, Proposition 2.6B].

$$
\operatorname{dim}_{k} \mathrm{H}^{i}\left(\Sigma_{d}, Y^{\lambda}\right)=\left[\mathrm{H}_{i}\left(\Sigma_{d}, V^{\otimes d}\right): L(\lambda)\right], i \geq 0 .
$$

Theorem 2.1 indicates that determining the simple constituents of $\mathrm{H}_{*}\left(\Sigma_{d}, V^{\otimes d}\right)$ as a graded $G$-module allows one to calculate Young module cohomology in all degrees. It turned out to be easier to study this for all $d$ simultaneously, using methods from algebraic topology. In [Cohen et al. 2010, Theorem 8.1.4] the algebra $\oplus_{d \geq 0} \mathrm{H}_{*}\left(\Sigma_{d}, V^{\otimes d}\right)$ is described as a $G$-module. It is a polynomial algebra, tensored with an exterior algebra if $p$ is odd. Each generator belongs to a certain $G$-module direct summand, and this summand belongs to $\mathrm{H}_{i}\left(\Sigma_{d}, V^{\otimes d}\right)$ for a particular $i$ and $d$.

The $G$-modules that occur are described below. For a $G$-module $M$ let $M^{(a)}$ denote the $a$-th Frobenius twist of $M$ (see [Jantzen 2003, p. 132]), and let $S^{a}(M)$ and $\Lambda^{a}(M)$ denote respectively the $a$-th symmetric and exterior power of $M$. 
Theorem 2.2 [Cohen et al. 2010, Corollary 8.2.1]. In characteristic two, the $G$ module $\mathrm{H}_{*}\left(\Sigma_{d}, V^{\otimes d}\right)$ is a direct sum of modules of the form

$$
S^{a_{0}}(V) \otimes S^{a_{1}}\left(V^{\left(c_{1}\right)}\right) \otimes \cdots \otimes S^{a_{s}}\left(V^{\left(c_{s}\right)}\right)
$$

where each $a_{i} \geq 0, c_{i}>0$ and $d=a_{0}+\sum_{j=1}^{s} a_{j} 2^{c_{j}}$.

In odd characteristic the $G$-module $\mathrm{H}_{*}\left(\Sigma_{d}, V^{\otimes d}\right)$ is a direct sum of modules of the form

$$
S^{a_{0}}(V) \otimes S^{a_{1}}\left(V^{\left(c_{1}\right)}\right) \otimes \cdots \otimes S^{a_{s}}\left(V^{\left(c_{s}\right)}\right) \otimes \Lambda^{d_{1}}\left(V^{\left(e_{1}\right)}\right) \otimes \cdots \otimes \Lambda^{d_{t}}\left(V^{\left(e_{t}\right)}\right)
$$

where each $a_{i} \geq 0$, each $c_{i}, d_{i}, e_{i}>0$ and where $d=a_{0}+\sum_{j=1}^{s} a_{j} p^{c_{j}}+\sum_{j=1}^{t} d_{j} p^{e_{j}}$.

Each summand in (2-1) or (2-2) occurs in $\mathrm{H}_{i}\left(\Sigma_{d}, V^{\otimes d}\right)$ for a single value of $d$ but for infinitely many different degrees $i$, for a description see Theorem 8.1.4 in [Cohen et al. 2010] or the special cases below, which are all we will use. To compute a particular $\mathrm{H}^{i}\left(\Sigma_{d}, Y^{\lambda}\right)$ one must first determine the (finitely many) summands which contribute to this $d$ and $i$, and then compute the multiplicities of $L(\lambda)$ in each summand. In the next section we will let $p=2$ and make a strategic choice for $\lambda$. For these $\lambda$ we can determine precisely the summand (2-1) of smallest degree which contains $L(\lambda)$ as a composition factor, and thus determine the initial vanishing range. In Section 4 we use these computations to produce Young modules with very large gaps in cohomology. In the final section we discuss the situation in odd characteristic, and present a few open problems.

\section{Initial vanishing ranges in characteristic two}

In this section assume $p=2$. Notation such as $\left(2^{3}, 1^{2}\right)$ will be shorthand for the partition $(2,2,2,1,1)$, not the partition $(8,1)$. It is clear from $(2-1)$ that understanding the composition factors of $S^{a}(V)$ is necessary for computing Young module cohomology (but not sufficient, as one must also decompose the tensor products).

Fortunately, Doty [1985] has determined the entire submodule structure for $S^{a}(V)$. The composition factors all occur with multiplicity at most one, and have a particularly nice form in characteristic two:

Proposition 3.1 [Doty 1985]. (See also [Cohen et al. 2010, Proposition 12.2.1].) Let $\lambda \vdash s$ have a 2-adic expansion

$$
\lambda=\sum_{i=0}^{m} 2^{i} \lambda_{(i)}
$$

where each $\lambda_{(i)}$ is 2-restricted. Then $L(\lambda)$ is a constituent of $S^{s}(V)$ if and only if each $\lambda_{(i)}$ is of the form $\left(1^{a_{i}}\right)$ for $a_{i} \geq 0$. 
Let $\mu=\left(\mu_{1}, \mu_{2}, \ldots, \mu_{r}\right) \vdash d$ be 2-restricted. Set $\mu^{\prime}=\left(\left(\mu^{\prime}\right)_{1},\left(\mu^{\prime}\right)_{2}, \ldots,\left(\mu^{\prime}\right)_{\mu_{1}}\right)$. We will compute the first $i$ such that $\mathrm{H}^{i}\left(\Sigma_{2 d}, Y^{2 \mu}\right)$ is nonzero, and see that a particular such $\mu$ will maximize the initial vanishing range.

Since $\mu$ is 2 -restricted, the 2 -adic expansion of $2 \mu$ is just $2 \mu$. So Steinberg's tensor product theorem (STPT) [Jantzen 2003, II.3.17] implies the summands from (2-1) with any $c_{i}>1$ do not have $L(2 \mu)$ as a composition factor. So to compute $\mathrm{H}^{i}\left(\Sigma_{2 d}, Y^{2 \mu}\right)$ we must determine the multiplicity of $L(2 \mu)$ in summands of the form

$$
S^{a}(V) \otimes S^{a_{1}}\left(V^{(1)}\right) \otimes \cdots \otimes S^{a_{s}}\left(V^{(1)}\right) \cong S^{a}(V) \otimes S^{\tau}\left(V^{(1)}\right)
$$

where we can assume without loss that $a_{i} \geq a_{i+1}$, so $\tau=\left(a_{1}, a_{2}, \ldots, a_{s}\right) \vdash d-\frac{a}{2}$.

Analysis just as in Section 10 of [Cohen et al. 2010] shows that a summand of the form (3-1) corresponds to monomials in the polynomial algebra of the form

$$
v^{a} \cdot Q_{i_{1}}^{a_{1}}(v) \cdots Q_{i_{s}}^{a_{s}}(v)
$$

for distinct $i_{t}$. By [Cohen et al. 2010, Theorem 8.1.4(a)], such a summand contributes to the cohomology in degree $a_{1} i_{1}+a_{2} i_{2}+\cdots+a_{s} i_{s}$. To determine the smallest $i$ with $\mathrm{H}^{i}\left(\Sigma_{2 d}, Y^{2 \mu}\right) \neq 0$ we must first determine which modules (3-1) contain $L(2 \mu)$ as a composition factor. Then for each we must determine the smallest possible corresponding degree where the summand can occur. Our assumption on $\mu$ limits how $L(2 \mu)$ can arise as a composition factor in (3-1):

Proposition 3.2. Let $2 \mu \vdash 2 d$ where $\mu$ is 2-restricted. Then $\mathrm{H}^{i}\left(\Sigma_{2 d}, Y^{2 \mu}\right) \neq 0$ if and only if there exists an integer $a \geq 0$, a partition $\tau=\left(a_{1}, a_{2}, \ldots, a_{s}\right) \vdash d-a$ and integers $\left\{i_{t}>0\right\}$ such that

(i) $i=a_{1} i_{1}+a_{2} i_{2}+\cdots+a_{s} i_{s}$,

(ii)

$$
\left[L\left(2^{a}\right) \otimes L\left(2^{a_{1}}\right) \otimes \cdots \otimes L\left(2^{a_{s}}\right): L(2 \mu)\right] \neq 0 .
$$

Proof. By Proposition 3.1 and the STPT, the composition factors of $S^{m}(V)$ are all of the form

$$
L\left(1^{c_{0}}\right) \otimes L\left(2^{c_{1}}\right) \otimes L\left(4^{c_{2}}\right) \otimes \cdots
$$

But $2 \mu$ is its own 2-adic expansion, so any $L(2 \mu)$ occurring in (3-1) must arise as in part (2) by the STPT. The corresponding degree $i$ follows from [Cohen et al. 2010, Theorem 8.1.4(a)].

Notice that the "if" part of the preceding result did not require $\mu$ be 2-restricted, a fact we will need later.

Now we want to find the smallest degree $i$ where the cohomology $\mathrm{H}^{i}\left(\Sigma_{2 d}, Y^{2 \mu}\right)$ is nonzero. Since $a_{1} \geq a_{2} \geq \cdots$, it is clear from Proposition 3.2(1) that we should 
choose $i_{t}=t$ to minimize the degree $i$. The smallest nonzero degree is given in terms of the following function on partitions. Let $\rho=\left(\rho_{1}, \rho_{2}, \ldots \rho_{s}\right) \vdash d$. Define

$$
x(\rho)=\sum_{l=1}^{s}(l-1) \rho_{l} .
$$

The following easy lemma is left to the reader:

Lemma 3.3. Suppose $\lambda \unrhd \mu$. Then $x(\lambda) \leq x(\mu)$. If $\lambda \neq \mu$ the inequality is strict.

We can now determine the first nonvanishing degree for $\mathrm{H}^{*}\left(\Sigma_{2 d}, Y^{2 \mu}\right)$.

Theorem 3.4. Let $\mu \vdash d$ be arbitrary. Then:

(i) $\mathrm{H}^{x\left(\mu^{\prime}\right)}\left(\Sigma_{2 d}, Y^{2 \mu}\right) \neq 0$.

(ii) If $\mu$ is 2-restricted, then

$$
\operatorname{dim} H^{i}\left(\Sigma_{2 d}, Y^{2 \mu}\right)= \begin{cases}0 & \text { for } 0 \leq i<x\left(\mu^{\prime}\right), \\ 1 & \text { for } i=x\left(\mu^{\prime}\right) .\end{cases}
$$

Proof. For convenience let $\tau=\mu^{\prime}$. Observe that

$$
\mu=\left(1^{\tau_{1}}\right)+\left(1^{\tau_{2}}\right)+\cdots+\left(1^{\tau_{\mu_{1}}}\right) .
$$

Then $L\left(2^{\tau_{1}}\right) \otimes L\left(2^{\tau_{2}}\right) \otimes \cdots \otimes L\left(2^{\tau_{\mu_{1}}}\right)$ has highest weight $2 \mu$ with multiplicity one, so

$$
\left[L\left(2^{\tau_{1}}\right) \otimes L\left(2^{\tau_{2}}\right) \otimes \cdots \otimes L\left(2^{\tau_{\mu_{1}}}\right): L(2 \mu)\right]=1
$$

Thus

$$
\left[S^{2 \tau_{1}}(V) \otimes S^{\tau_{2}}\left(V^{(1)}\right) \otimes S^{\tau_{3}}\left(V^{(1)}\right) \cdots \otimes S^{\tau_{\mu_{1}}}\left(V^{(1)}\right): L(2 \mu)\right] \geq 1 .
$$

Choosing $a=\tau_{1}$ and $i_{t}=t$, the proof of Proposition 3.2 tells us that

$$
\mathrm{H}^{x\left(\mu^{\prime}\right)}\left(\Sigma_{2 d}, Y^{2 \mu}\right) \neq 0
$$

(The "if" part did not require $\mu$ be 2-restricted.)

Now suppose further that $\mu$ is 2-restricted, and consider Proposition 3.2. Suppose

$$
\left[L\left(2^{a}\right) \otimes L\left(2^{a_{1}}\right) \otimes \cdots \otimes L\left(2^{a_{s}}\right): L(2 \mu)\right] \neq 0 .
$$

In order to minimize the degree $i$ it is clear from Proposition 3.2(2) that we may assume $a \geq a_{1} \geq a_{2} \geq \cdots \geq a_{s}$. Then $\rho:=\left(a, a_{1}, a_{2}, \ldots, a_{s}\right) \vdash d$, and by Proposition 3.2(1), the corresponding cohomological degree is $x(\rho)$. Since $L\left(2^{a}\right) \otimes L\left(2^{a_{1}}\right) \otimes \cdots \otimes L\left(2^{a_{s}}\right)$ has highest weight $2 \rho^{\prime}$ then $\rho^{\prime} \unrhd \mu$, and thus $\mu^{\prime} \unrhd \rho$. When $\rho=\mu^{\prime}$ we get a single copy of $L(2 \mu)$ as above, contributing to degree $x\left(\mu^{\prime}\right)$. Otherwise $\mu^{\prime} \triangleright \rho$. Then Lemma 3.3 implies $x\left(\mu^{\prime}\right)<x(\rho)$, so $x\left(\mu^{\prime}\right)$ is the smallest degree with nonzero cohomology. So the cohomology is one-dimensional in degree $x\left(\mu^{\prime}\right)$ and zero in smaller degrees. 


\section{Mind the gap}

In this section we apply Theorem 3.4 to find large gaps in cohomology. For comparison we first compute the smallest currently known $r\left(\Sigma_{d}\right)$ which satisfies Theorem 1.1.

A faithful complex representation of a group $G$ gives rise to an embedding into a compact unitary group $G \hookrightarrow U(n)$. The cohomology of the classifying space $B U(n)$ is a polynomial ring on generators in degrees $2,4,6, \ldots, 2 n$ (see [Benson 1998, Section 2.6]). The value of $r$ coming from these generators by the construction in [Benson et al. 1990] is $1+3+5+\cdots+(2 n-1)=n^{2}$. Thus if $G$ has a faithful representation of dimension $n$, one can take $r=r(G)=n^{2}$ in Theorem 1.1, see [Benson 1998, Sections 5.14-15] for details.

The smallest faithful irreducible $\mathbb{C} \Sigma_{d}$ module is $d-1$ dimensional, so one can take $r\left(\Sigma_{d}\right)=(d-1)^{2}$, and this is the smallest known bound. To find Young modules with large vanishing ranges, Theorem 3.4(2) suggests finding $p$-restricted $\mu$ with $x\left(\mu^{\prime}\right)$ as large as possible. In this section we show careful choice of Young module can realize gaps on the order of $\frac{1}{3} d^{3 / 2}$.

Fix $n \geq 1$ and define $\rho_{n}=(n, n-1, n-2, \ldots, 2,1) \vdash \frac{1}{2}\left(n^{2}+n\right)$. Notice that

$$
\rho_{n}=\left(\rho_{n}\right)^{\prime}=\left(1^{n}\right)+\left(1^{n-1}\right)+\cdots+\left(1^{2}\right)+(1) .
$$

One easily computes that

$$
x\left(\rho_{n}\right)=\frac{n^{3}-n}{6} .
$$

Proposition 4.1. Let $p=2$ and $\rho_{n} \vdash\left(n^{2}+n\right) / 2$ be as above. Then:

$$
\operatorname{dim} \hat{\mathrm{H}}^{i}\left(\Sigma_{n^{2}+n}, Y^{2 \rho_{n}}\right)= \begin{cases}0 & \text { for }-\frac{1}{6}\left(n^{3}-n\right)<i<\frac{1}{6}\left(n^{3}-n\right), \\ 1 & \text { for } i= \pm \frac{1}{6}\left(n^{3}-n\right) .\end{cases}
$$

Proof. Since $\rho_{n}$ is 2-restricted, we can apply Theorem 3.4(2) and (4-1). The extension to negative degrees comes from Tate duality, using the fact that Young modules are self-dual.

Proposition 4.1 shows that for $d=n^{2}+n$, the best possible $r\left(\Sigma_{d}\right)$ is at least $\frac{1}{3}\left(n^{3}-n\right)$.

Example 4.2. Let $\lambda=(28,26,24, \ldots, 6,4,2) \vdash 210$. Then

$$
\mathrm{H}^{i}\left(\Sigma_{210}, Y^{\lambda}\right)= \begin{cases}0 & \text { if }-455<i<455 \\ k & \text { if } i=455\end{cases}
$$

It follows from [Hemmer and Nakano 2002, Theorem 3.3.2] that the $Y^{\lambda}$ in Example 4.2 has complexity 105 , the maximum possible among $\Sigma_{210}$-modules. This means the dimension of the module $P_{i}$ in the minimal projective resolution 
$P_{*} \rightarrow k$ of the trivial module grows like a polynomial of degree 104 in $i$. However it is not until $P_{455}$ that the projective cover $P(k)$ makes its first appearance!

Remark 4.3. Proposition 4.1 applies to $\Sigma_{2 d}$ where $d$ is a triangular number $T(n)=$ $\frac{n^{2}+n}{2}$. For arbitrary $d$ one can still choose a 2-restricted $\mu$ maximizing $x\left(\mu^{\prime}\right)$ in a similar way. Write $d=T(n)+a$ for $0 \leq a<n+1$ and choose

$$
\mu=(n, n-1, \ldots, a+1, a, a, a-1, a-2, \ldots, 2,1) .
$$

One still has $x\left((2 \mu)^{\prime}\right)$ asymptotic to a constant times $n^{3}$.

So there is a constant $c$ so that for arbitrary $d$ we can obtain Young modules in characteristic two with cohomology vanishing for the first $c d^{3 / 2}$ degrees.

\section{Odd primes and further directions}

Since Theorem 1.1 gives a bound $r(G)$ independent of the characteristic, we have focused on $p=2$ which gives the cleanest results. For an arbitrary prime one can still achieve gaps that are a constant times $d^{3 / 2}$ in length, using $\mu=p(p-1) \rho$, although the answer is messier, and involves polynomials in $p$. For example the nice compact form for $x\left(\rho_{n}\right)$ in (4-1) becomes replaced by

$$
(p-1)[n(2 p-3)+(n-1)(4 p-5)+\cdots+1(2 n(p-1)-1)] .
$$

The corresponding result, which we state without proof, is this:

Proposition 5.1. Let $d=\frac{1}{2} p(p-1)\left(n^{2}+n\right)$. Let $\mu=p(p-1) \rho_{n} \vdash d$. Then there is a constant $c(p)$ and a polynomial $p(n)=c(p) n^{3}+a n^{2}+b n$ such that

$$
\mathrm{H}^{i}\left(\Sigma_{d}, Y^{\mu}\right)=0 \text { if }-p(n)<i<p(n)
$$

So once again we have find an $r\left(\Sigma_{d}\right)$ asymptotic to a constant times $d^{3 / 2}$. The function $c(p)$ is decreasing, so the best estimates for $r\left(\Sigma_{d}\right)$ come from the $p=2$ case. This might lead one to make a wild conjecture:

Conjecture 5.2. Let $d=\frac{1}{2} p(p-1)\left(n^{2}+n\right)$. Let $\mu=p(p-1) \rho_{n} \vdash d$. Among all $\Sigma_{d}$ modules in the principal block with nonvanishing cohomology, the Young module $Y^{\mu}$ has the largest gap in cohomology, and thus determines the best possible $r$ in Theorem 1.1. For $d$ not of this form, a similar choice, in the spirit of (4-2), for $\mu$ achieves the maximal gap.

There are many problems which remain, although it isn't clear one should expect nice answers to any of them. For example one might find the smallest positive $i$ with $\mathrm{H}^{i}\left(\Sigma_{d}, Y^{\lambda}\right) \neq 0$. The corresponding problem for simple modules is a subject of active research, for example for groups of Lie type. A first step would be to generalize Doty's work from the $\mu=(d)$ case to something more general: 
Problem 5.3. Given $\lambda \vdash d$, find the maximal $\mu \vdash d$ such that $\left[S^{\mu}(V): L(\lambda)\right] \neq 0$.

Determining the $\lambda$ for which $\mu=(d)$ is just Doty's result on the composition factors of $S^{d}(V)$. At the opposite extreme, such a $\mu$ always exists, because $S^{\left(1^{d}\right)}(V) \cong V^{\otimes d}$ and each $L(\mu)$ occurs as a composition factor of $V^{\otimes d}$.

Finally we observe that the partition $\mu$ appearing in Proposition 5.1 is just the twist of the Steinberg weight (see [Jantzen 2003, p. 199]), but there seems to be no representation-theoretic interpretation of this fact.

\section{References}

[Benson 1995] D. J. Benson, "Cohomology of modules in the principal block of a finite group", New York J. Math. 1 (1995), 196-205. MR 96h:20095 Zbl 0879.20004

[Benson 1998] D. J. Benson, Representations and cohomology, II: Cohomology of groups and modules, 2nd ed., Cambridge Studies in Advanced Mathematics 31, Cambridge University Press, 1998. MR 99f:20001b Zbl 0908.20002

[Benson et al. 1990] D. J. Benson, J. F. Carlson, and G. R. Robinson, "On the vanishing of group cohomology", J. Algebra 131:1 (1990), 40-73. MR 91c:20073 Zbl 0697.20043

[Cohen et al. 2010] F. R. Cohen, D. J. Hemmer, and D. K. Nakano, "On the cohomology of Young modules for the symmetric group", Adv. Math. 224:4 (2010), 1419-1461. MR 2011f:20118 Zbl 1206.20057

[Doty 1985] S. R. Doty, "The submodule structure of certain Weyl modules for groups of type $A_{n}$ ", J. Algebra 95:2 (1985), 373-383. MR 86j:20035 Zbl 0577.20031

[Doty et al. 2004] S. R. Doty, K. Erdmann, and D. K. Nakano, "Extensions of modules over Schur algebras, symmetric groups and Hecke algebras", Algebr. Represent. Theory 7:1 (2004), 67-100. MR 2005e:20004 Zbl 1084.20004

[Hemmer and Nakano 2002] D. J. Hemmer and D. K. Nakano, "Support varieties for modules over symmetric groups", J. Algebra 254:2 (2002), 422-440. MR 2003k:20013 Zbl 1028.20009

[Jantzen 2003] J. C. Jantzen, Representations of algebraic groups, 2nd ed., Mathematical Surveys and Monographs 107, American Mathematical Society, Providence, RI, 2003. MR 2004h:20061 Zbl 1034.20041

[Martin 1993] S. Martin, Schur algebras and representation theory, Cambridge Tracts in Mathematics 112, Cambridge University Press, 1993. MR 95f:20071 Zbl 0802.20011

Communicated by David Benson

Received 2011-06-20 Accepted 2011-08-13

dhemmer@math.buffalo.edu Department of Mathematics, University at Buffalo, SUNY, College of Arts and Sciences, The State University of New York, Buffalo, NY 14260-2900, United States http://math.buffalo.edu/ dhemmer/ 


\section{Algebra \& Number Theory}

msp.berkeley.edu/ant

\section{EDITORS}

\author{
MANAGING EDITOR \\ Bjorn Poonen \\ Massachusetts Institute of Technology \\ Cambridge, USA
}

\author{
EDITORIAL BOARD CHAIR \\ David Eisenbud \\ University of California \\ Berkeley, USA
}

\section{BOARD OF EDITORS}

$\begin{aligned} \text { Georgia Benkart } & \text { University of Wisconsin, Madison, USA } \\ \text { Dave Benson } & \text { University of Aberdeen, Scotland } \\ \text { Richard E. Borcherds } & \text { University of California, Berkeley, USA } \\ \text { John H. Coates } & \text { University of Cambridge, UK } \\ \text { J-L. Colliot-Thélène } & \text { CNRS, Université Paris-Sud, France } \\ \text { Brian D. Conrad } & \text { University of Michigan, USA } \\ \text { Hélène Esnault } & \text { Universität Duisburg-Essen, Germany } \\ \text { Hubert Flenner } & \text { Ruhr-Universität, Germany } \\ \text { Edward Frenkel } & \text { University of California, Berkeley, USA } \\ \text { Andrew Granville } & \text { Université de Montréal, Canada } \\ \text { Joseph Gubeladze } & \text { San Francisco State University, USA } \\ \text { Ehud Hrushovski } & \text { Hebrew University, Israel } \\ \text { Craig Huneke } & \text { University of Kansas, USA } \\ \text { Mikhail Kapranov } & \text { Yale University, USA } \\ \text { Yujiro Kawamata } & \text { University of Tokyo, Japan } \\ \text { János Kollár } & \text { Princeton University, USA } \\ \text { Yuri Manin } & \text { Northwestern University, USA } \\ \text { Barry Mazur } & \text { Harvard University, USA } \\ \text { Philippe Michel } & \text { École Polytechnique Fédérale de Lausanne } \\ \text { Susan Montgomery } & \text { University of Southern California, USA }\end{aligned}$

Shigefumi Mori

Raman Parimala

Jonathan Pila

Victor Reiner

Karl Rubin

Peter Sarnak

Joseph H. Silverman

Michael Singer

Ronald Solomon

Vasudevan Srinivas

J. Toby Stafford

Bernd Sturmfels

Richard Taylor

Ravi Vakil

Michel van den Bergh

Marie-France Vignéras

Kei-Ichi Watanabe

Andrei Zelevinsky

Efim Zelmanov
RIMS, Kyoto University, Japan

Emory University, USA

University of Oxford, UK

University of Minnesota, USA

University of California, Irvine, USA

Princeton University, USA

Brown University, USA

North Carolina State University, USA

Ohio State University, USA

Tata Inst. of Fund. Research, India

University of Michigan, USA

University of California, Berkeley, USA

Harvard University, USA

Stanford University, USA

Hasselt University, Belgium

Université Paris VII, France

Nihon University, Japan

Northeastern University, USA

University of California, San Diego, USA

\section{PRODUCTION}

contact@msp.org

Silvio Levy, Scientific Editor

See inside back cover or www.jant.org for submission instructions.

The subscription price for 2012 is US \$175/year for the electronic version, and \$275/year ( $\$ 40$ shipping outside the US) for print and electronic. Subscriptions, requests for back issues from the last three years and changes of subscribers address should be sent to Mathematical Sciences Publishers, Department of Mathematics, University of California, Berkeley, CA 94720-3840, USA.

Algebra \& Number Theory (ISSN 1937-0652) at Mathematical Sciences Publishers, Department of Mathematics, University of California, Berkeley, CA 94720-3840 is published continuously online. Periodical rate postage paid at Berkeley, CA 94704, and additional mailing offices.

ANT peer review and production are managed by EditFLOW ${ }^{\circledR}$ from Mathematical Sciences Publishers.

PUBLISHED BY

mathematical sciences publishers

http://msp.org/

A NON-PROFIT CORPORATION

Typeset in IATEX

Copyright @2012 by Mathematical Sciences Publishers 


\section{Algebra \& Number Theory}

Volume $6 \quad$ No. $4 \quad 2012$

Spherical varieties and integral representations of $L$-functions

YIANNIS SAKELLARIDIS

Nonuniruledness results for spaces of rational curves in hypersurfaces

ROYA BEHESHTI

Degeneracy of triality-symmetric morphisms

DAVE ANDERSON

Multi-Frey $\mathbb{Q}$-curves and the Diophantine equation $a^{2}+b^{6}=c^{n}$

Michael A. BenNetT and IMIN CHEN

Detaching embedded points

DAWEI CHEN and SCOTT NOLLET

Moduli of Galois $p$-covers in mixed characteristics

Dan ABRAMOVICH and MatThiEU Romagny

Block components of the Lie module for the symmetric group ROGER M. BRYANT and KARIN ERDMANN

Basepoint-free theorems: saturation, b-divisors, and canonical bundle formula OSAMU FUJINO

Realizing large gaps in cohomology for symmetric group modules DAVID J. HEMMER 